\title{
Constructal design in the cooling and hydraulic performance of tube heat sinks
}

\author{
Sheng Chen ${ }^{\mathrm{a}}$, Antonio F. Miguel ${ }^{\mathrm{b}, *}$, Murat Aydin ${ }^{\mathrm{c}}$

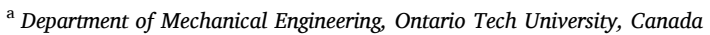 \\ ${ }^{\mathrm{b}}$ Department of Physics, University of Evora, Portugal \\ ${ }^{\mathrm{c}}$ Department of Automotive and Mechatronics Engineering, Ontario Tech University, Canada
}

\section{A R T I C L E I N F O}

\section{Keywords:}

Cooling flow design

Heat dissipation

Temperature distribution

Flow resistance

Hydrothermal performance

Constructal law

Electric vehicle and battery pack

\begin{abstract}
A B S T R A C T
Liquid-cooled hot plates require effective thermal management which can be accomplished by implementing appropriate cooling architectures. This investigation is about high heat-generating systems and reports the performance of heated plates with various cooling flow configurations: Serpentine flow, parallel tubes flow, and two tree-shape flow systems. Single and double objectives with size constraint are studied to obtain the best design. The influence of fluid inlet position is also studied. The results are presented in terms of hydraulic and thermal characteristics for plates that is subject to a constant heat flux, and contain tubes with fixed total lateral area for cooling. A 3D numerical study is conducted to analyze the performance of these systems for Reynolds numbers ranging from 1000 to 33,000. The results obtained include temperatures of the plate, uniformity of temperature of the plate, fluid flow resistance and heat dissipation. Dimensionless parameters are identified to characterize the thermal performance, hydraulic performance, and hydrothermal performance as a function of the Reynolds number. The results show that a tree-shaped flow network of tubes inserted in the plate with inlet placed at the center of the plate presents the best cooling performance, with more cooling capacity and less flow resistance (and pumping power).
\end{abstract}

\section{Introduction}

Heat dissipation is important in various engineering and industrial applications such as electronic cooling devices, battery thermal management, solar thermal collector systems, etc. The continuous reduction of size of the systems combined with an increase in the power of the systems requires the dissipation of large amount of heat, especially in high heat-generating devices. An appropriate thermal management is essential for these systems to ensure performance, reliability, lifespan, and safety.

The increasing density of transistors in electronic components requires an efficient heat dissipation [1]. Management of electric vehicle power batteries are essential for mileage, self-discharge, safety, and useful life of these vehicles [2]. Heat dissipation affects battery charge and discharge performances, aging, and degradation [3]. Photovoltaic thermal collectors show an enormous potential due to the possibility of generating electrical and thermal energy [4]. Cooling tubes placed behind the photovoltaic modules can be used to supply hot water and heating, increasing overall efficiency [5]. In these and other applications, tubes are used to remove heat by means a working fluid that flows inside the cooling network. Therefore, the performance of these tubes is a key parameter for an efficient utilization of the system.

To ensure the reliability of the electronic systems, with a suitable heat dissipation from the electronic components, microtubes heat sinks area is a promising solution [6]. Serpentine tube heat sink design is described as a good option to address the problem of thermal management [7]. Wang et al. [8] also studied cooling system designs composed by parallel tubes. They concluded that the performance of heat dissipation depends on the number of tubes, and the inlet mass flow rate.

Leng et al. [9] reported that a truncated channel design reduced the maximum difference of peak temperature by $36.7 \%$ compared to a double-layered parallel straight channel heat sink.

Senn and Poulikakos [10] and Chen et al. [11] concluded that tree network heat sinks provide better heat transfer capacity and the pressure loss than those of serpentine design when the heat transfer area is constant, under laminar flow regime. Recently, Mosa et al. [12,13] studied the influence of serpentine and branching flow designs on the performance of suspended radiant cooling panels in laminar flow. They

\footnotetext{
* Corresponding author.

E-mail address: afm@uevora.pt (A.F. Miguel).
} 
conclude that the cooling performance increases with Reynolds number and branching design has the best performance.

The effect of the inlet and outlet layout of a Y-shaped microchannel heat sink is investigated by Lu and Zhang [14]. They concluded that more uniform temperature distribution is obtained by replacing the layout of inlet and outlet on the same side with opposite configuration. A network of parallel cooling tubes is preferred in photovoltaic thermal collectors due to low pressure drop [15].

Comprehensive reviews of the literature on the effect of geometry on cooling performance are provided by Joshi and Dhoble [16], and Narendran et al. [17]. These last authors reviewed the characteristics of straight channel, serpentines, clover channel, and branched networks.

This study is devoted to the design of network of tubes for cooling high heat-generating systems from a Constructal law perspective [18]. The aim is to provide a better hydrothermal performance by an easy access for both the heat that is transferred to the fluid pumped through the tubes, and the fluid flow (low power dissipation). Designs include a serpentine configuration, parallel flow tube system, and two tree-shaped flow networks of tubes. To compare these designs, it is required to include size constraints to account for the fact that space is limited. For a plate of constant size, the performance of different cooling designs is evaluated respecting the surface area -limiting requirement. To address this, a 3D numerical study is carried out to find out the influence of geometry and flow on both cooling performance and flow resistance, the uniformity of temperatures on the plate.

\section{Design models}

The schematic representation of cooling systems inserted in a panel that generates a constant amount of heat is represented in Fig. 1. An aluminum cooling plate is used in this study with a constant volume (1.6 $\mathrm{m}$ long, $1.2 \mathrm{~m}$ wide, and $0.04 \mathrm{~m}$ thick). The inlet and outlet diameter of tubes are $30 \mathrm{~mm}$ for the serpentine configuration (Fig. 1a), $32.58 \mathrm{~mm}$ for the parallel configuration (Fig. 1b), and $32.6 \mathrm{~mm}$ for the tree-shaped configuration (Fig. 1c). For the tree-shaped flow configuration (inlet at center), the inlet diameter (Fig. 1d) is $40 \mathrm{~mm}$ and the outlet diameters varied between $25 \mathrm{~mm}$ and $4 \mathrm{~mm}$. Both the volume of the panel and the total lateral surface area of tubes are fixed (constants), with the exception of the tree configuration depicted at Fig. 1d which has a total surface area of the tubes $18 \%$ smaller than the other configurations. This difference was due to the difficulty experienced in realizing the 3D structure of the configuration. The working fluid that circulates inside the tubes is ethylene-glycol mixed with water and the properties are described in Table 1.

As the heat loss to the surrounding environment is proportional to the body surface area of the plate [19], the total lateral surface area of the tubes is fixed as size constraint applied to the configuration of tubes (Fig. 1). Table 2 presents the sizes of tubes for the geometries studied numerically.

A parent tube dividing into two daughter branches (Y-shaped junc-
Table 1

Properties of the working fluid ethylene-glycol mixed with water.

\begin{tabular}{ll}
\hline Coolant properties $(T=300 \mathrm{~K})$ & \\
\hline Viscosity $(\mathrm{Pa} \cdot s)$ & 0.00315 \\
Conductivity $\left(\mathrm{Wm}^{-1} \mathrm{~K}^{-1}\right)$ & 0.419 \\
Specific Heat $\left(\mathrm{Jkg}^{-1} \mathrm{~K}^{-1}\right)$ & 3494 \\
Density $\left(\mathrm{kgm}^{-3}\right)$ & 1065 \\
\hline
\end{tabular}

Table 2

Sizes of geometry of tubes depicted at Fig. 1.

\begin{tabular}{lll}
\hline Flow configuration & Inlet diameter $(\mathrm{m})$ & Total lateral area of tubes $\left(\mathrm{m}^{2}\right)$ \\
\hline Serpentine & 0.03000 & 1.20 \\
Parallel tubes & 0.03258 & 1.20 \\
Tree-shaped (parent tube) & 0.03260 & 1.20 \\
Center Out & 0.04000 & 0.91 \\
\hline
\end{tabular}

tion) is the fundamental building block of the tree network [20]. The size of branched tubes designed to provide and easier access to flow can be obtained under different size constraints [20-24]. For a fixed lateral surface area of the tubes, the optimal size ratio between the two levels of branching and the optimal angle between daughter tubes are [20-23]

$\frac{D_{d}}{D_{p}}=2^{-\frac{2}{5}}, \frac{L_{d}}{L_{p}}=2^{-\frac{3}{5}} ; \theta_{p}=97^{0}$ (laminar regime)

$\frac{D_{d}}{D_{p}}=\frac{L_{d}}{L_{p}}=2^{-\frac{1}{2}} ; \theta_{p}=90^{0}$ (turbulent regime)

where $D_{p}$ is the diameter of the parent tube, $D_{d}$ is the diameter of the daughter tube, $L_{p}$ is the length of the parent tube, $L_{d}$ is the length of the daughter tube, and $\theta_{\mathrm{p}}$ is the optimal angle between daughter tubes.

Note that the geometry depicted at Fig. 1c is the design based on Eqs. (1) and (2) for laminar and turbulent regimes, respectively. However, the geometry shown at Fig. 1d is design only based on Eq. (1), and tested for both flow regimes, to evaluate its performance outside the regime for which it was built.

\subsection{Numerical study}

Simulations of fluid flow and heat transfer through the cooling configurations were performed using ANSYS-Fluent code [25]. For steady-state, Reynolds-averaged conservation equations for mass, momentum and energy are solved for velocity, pressure, and temperature distributions. A k- $\omega$ Shear Stress Transport (SST) turbulence model was selected, and the governing equations are summarized as follows:

Continuity equation:

$\frac{\partial}{\partial x_{i}}\left(\rho u_{i}\right)=0$

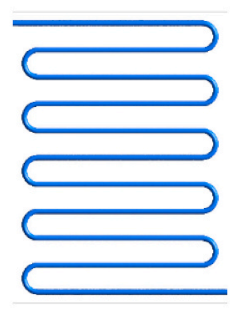

(a)

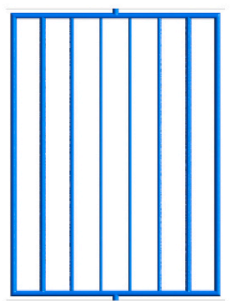

(b)

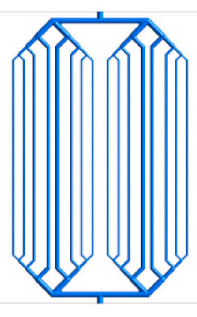

(c)

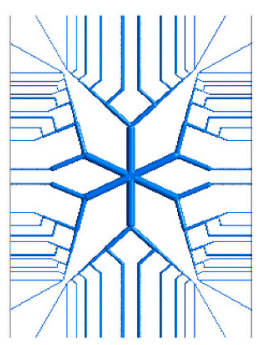

(d)

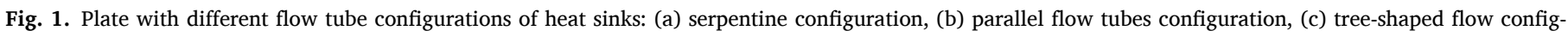
uration (inlet at edge), and (d) tree-shaped flow configuration (inlet at center). 
Momentum equation:

$\frac{\partial}{\partial x_{i}}\left(\rho u_{i} u_{j}\right)=-\frac{\partial p}{\partial x_{i}}+\frac{\partial}{\partial x_{j}}\left[\mu\left(\frac{\partial u_{i}}{\partial x_{j}}+\frac{\partial u_{j}}{\partial x_{i}}-\frac{2}{3} \delta_{i j} \frac{\partial u_{j}}{\partial x_{i}}\right)\right]+\frac{\partial}{\partial x_{j}}\left(-\rho \overline{u_{i}^{\prime} u_{j}^{\prime}}\right)$

Energy equation:

$c_{p} \frac{\partial}{\partial x_{i}}\left(\rho u_{i} T\right)=k \frac{\partial}{\partial x_{i}}\left(\frac{\partial T}{\partial x_{i}}\right)$

The Reynolds stress $-\rho \overline{u_{i}^{\prime} u_{j}^{\prime}}$ is modeled based on $k$ - $\omega$ model as

$\frac{\partial}{\partial t}(\rho k)+\frac{\partial}{\partial x_{i}}\left(\rho k u_{i}\right)=\frac{\partial}{\partial x_{j}}\left(\zeta_{k} \frac{\partial k}{\partial x_{j}}\right)+\widetilde{G}-Y_{k}+S_{k}$

$\frac{\partial}{\partial t}(\rho \omega)+\frac{\partial}{\partial x_{i}}\left(\rho \omega u_{i}\right)=\frac{\partial}{\partial x_{j}}\left(\zeta_{\omega} \frac{\partial k}{\partial x_{j}}\right)+G_{\omega}-Y_{k}+D_{\omega}+S_{k}$

where $\widetilde{G}$ is the generation of turbulence due to mean velocity gradients, $G_{\omega}$ is the generation of $\omega, \zeta_{k}$ and $\zeta_{\omega}$ are the diffusivity of $k$ and $\omega, Y_{k}$ and $\mathrm{Y}_{\omega}$ are the dissipation of $k$ and $\omega$ due to turbulence, $D_{\omega}$ is the crossdiffusion term, and $S_{k}$ and $S_{\omega}$ are user defined source terms if any. The constants used for the turbulent model are summarized in Table 3 .

\subsection{Numerical model}

All designs (Fig. 1) comply with the following boundary conditions. A uniform heat flux of $20 \mathrm{kWm}^{-2}$ is prescribed at the top side of the plate. At tube inlet, a constant mass flow rate and a fixed fluid temperature are prescribed. Atmospheric pressure was specified at the outlet. No-slip boundary condition is employed on the walls within the tubes. The operating conditions are summarized in Table 4.

Both coupled and SIMPLE algorithms are applied to solve pressurevelocity coupling. Second-order upwind scheme and second order central scheme are adopted to discretize the convective (inertial) and diffusion (viscous) terms of budget equations, respectively. The residual criteria for the continuity equation, and for momentum and energy equations are set to $10^{-5}$ and $10^{-7}$, respectively.

The influence of the grid resolution on the results is studied, and a mesh sensitivity analysis for all designs studied was performed based on the ASME criteria [23]. A mesh of poly-hexcore elements with near-wall refinements, 5 inflation layers in the fluid domain, and 5 layers in the solid domain is adopted. A typical mesh for the configurations can be seen in Fig. 2. The minimum numbers of element that satisfied the grid independency criterion are seen as of $8 \times 10^{5}$ to $4 \times 10^{6}$ elements depending on the geometry.

\section{Hydrothermal performance}

The performance of the designs (Fig. 1) is affected by the amount of heat removed but also by the power required to pump the working fluid. The best hydrothermal performance requires a high level of heat removed and a low flow resistance. To keep the plate at a safe temperature range and maintain a high hydrothermal performance a compromise between the fluid flow and the amount of heat removed should be

Table 3

Constants for Eqs. (6) and (7).

\begin{tabular}{ll}
\hline Alpha_inf & 1 \\
Beta*_inf & 0.52 \\
A1 & 0.31 \\
Beta_i (inner) & 0.075 \\
Beta_i (outer) & 0.0828 \\
TKE (inner) Prandtl \# & 1.176 \\
TKE (outer) Prandlt \# & 1 \\
SDR (inner) Prandlt \# & 2 \\
Energy Prandtl Number & 0.85 \\
Wall Prandlt Number & 0.85 \\
Production Limiter Clip Factor & 10 \\
\hline
\end{tabular}

Table 4

Operating conditions for the geometry of tubes depicted at Fig. 1.

\begin{tabular}{ll}
\hline Flow configurations & \\
\hline Heat flux density $\left(\mathrm{kW} / \mathrm{m}^{2}\right)$ & 20 \\
Inlet mass flow rate $(\mathrm{kg} / \mathrm{s})$ & $0.25,0.5,1.0,1.5,2.0,2.5$ \\
Initial inlet temperature $(\mathrm{K})$ & 300 \\
Outlet pressure $(\mathrm{Pa})$ & 0 \\
\hline
\end{tabular}

found.

The heat removed by the working fluid is given by

$Q_{\text {heat }}=\dot{m}_{f} c_{p . f} \Delta T_{f}$

and the resistance to flow of the working fluid can be written as

$R_{\text {fluid }}=\frac{\rho \Delta p}{\dot{m}_{f}}$

where $Q_{\text {heat }}$ is the heat removed by the working fluid, $R_{\text {fluid }}$ is the resistance to fluid flow, $\dot{m}_{f}$ is the mass flow rate of the working fluid, $\mathrm{c}_{\mathrm{p}, \mathrm{f}}$ is the specific heat at constant pressure of the working fluid, and $\Delta T_{f}$ is temperature difference between the working fluid at outlet and at inlet.

Eqs. (8) and (9) can be written in the following dimensionless form [24]

$N u=\frac{Q_{\text {heat }} D}{k_{f} A_{l s} T^{*}}$

$E u=\frac{\rho A_{\text {inlet }}^{2} \Delta p}{\dot{m}_{f}^{2}}$

with

$T^{*}=\frac{\left(T_{f, \text { outlet }}-T_{f, \text { inlet }}\right)}{\ln \left(\frac{\overline{T_{p}}-T_{f, \text { inlet }}}{\overline{T_{p}}-T_{f, \text { out }}}\right)}$

where $N u$ is the Nusselt number, $E u$ is the Euler number, D is the inlet tube diameter, $A_{l s}$ is the lateral surface area of the tubes, $A_{\text {inlet }}$ is the cross-sectional area of the inlet tube, $\mathrm{T}^{*}$ is the log mean temperature difference, $\overline{T_{p}}$ is the mean temperature of the plate, and $\mathrm{T}_{\mathrm{f} \text {,inlet }}$ and $\mathrm{T}_{\mathrm{f}}$, outlet are the temperatures of the working fluid at the inlet and at the outlet, respectively.

For a tree-shaped configuration of $n^{\text {th }}$ final branching levels, $A_{l s}$ is given by $[25,26]$

$A_{s l}=\pi D_{\text {inlet }} L_{\text {inlet }} \frac{1-\left[2\left(\frac{D_{d}}{D_{p}}\right)\left(\frac{L_{d}}{L_{p}}\right)\right]^{n+1}}{1-2\left(\frac{D_{d}}{D_{p}}\right)\left(\frac{L_{d}}{L_{p}}\right)}$

As mentioned before, in addition to the heat removed from the plate ( $\left.Q_{\text {heat }}\right)$, and the mean temperature of the plate $\left(\overline{T_{p}}\right)$, the pumping power (P) of a working fluid with specific properties (density $\rho$, viscosity $\mu$, and specific heat $c_{p . f}$ ) is of great importance because represents the cost to drive the fluid through the tubes. Let $\mathrm{P}, \mathrm{Q}_{\text {heat }}, \overline{T_{p}}, \rho, \mu$, and $\mathrm{c}_{\mathrm{p} . \mathrm{f}}$ be dimensional variables that are physically relevant to hydrothermal performance. Invoking the Vaschy-Riabouchinsky-Buckingham's П-theorem leads to the hydrothermal performance $\zeta$

$\zeta=\frac{\mu^{2}}{c_{p, f} \rho^{2} A_{p}} \frac{Q_{\text {heat }}}{\bar{T}_{p} P}$

with

$P=\frac{\dot{m}_{f} \Delta p}{\rho}$ 


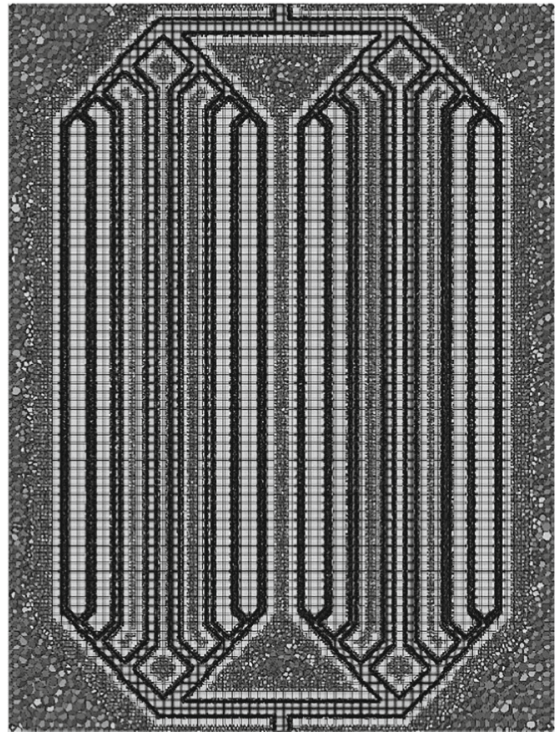

(a)

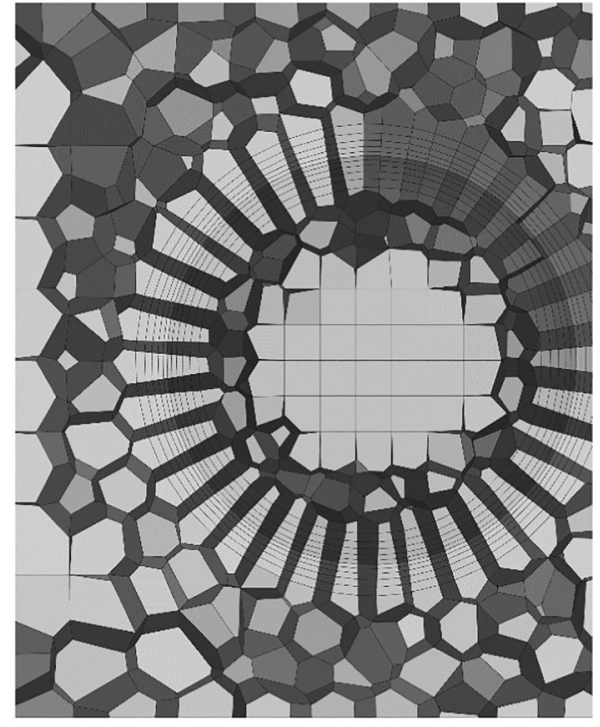

(b)

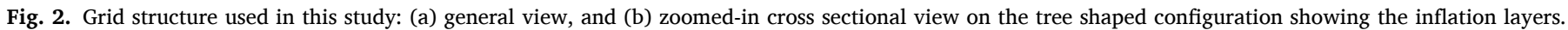

here $\zeta$ represents the hydrothermal performance defined as the thermal upgrading to the flow resistance penalty during the cooling operation, and $A_{p}$ is the plate surface area.

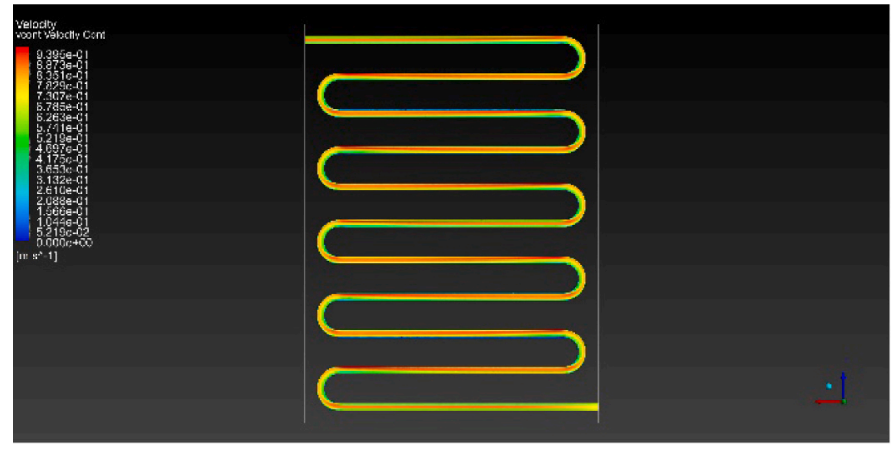

(a)

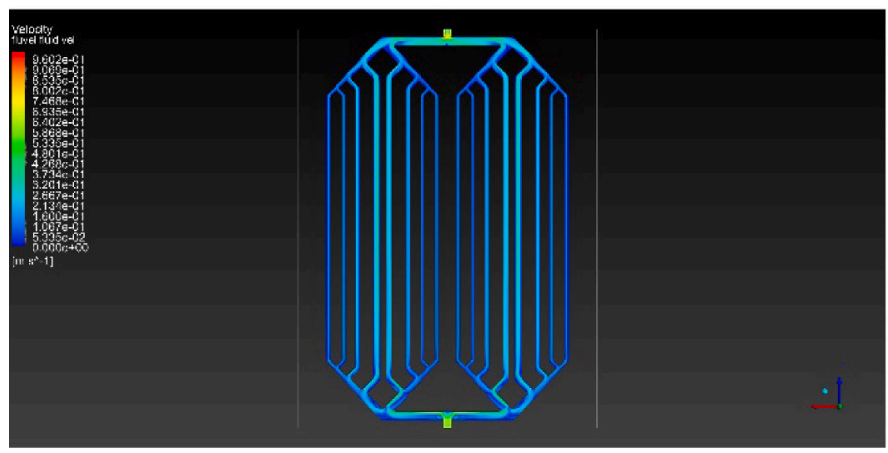

(c)

\section{Results}

4.1. Temperature profiles in the plates and fluid flow characteristics

The flow development within the tubes is shown by depicting the distributions of velocities (Figs. 3 and 4). Flow separation can be seen at the sharp corners in the parallel and both tree shaped designs. Due to the

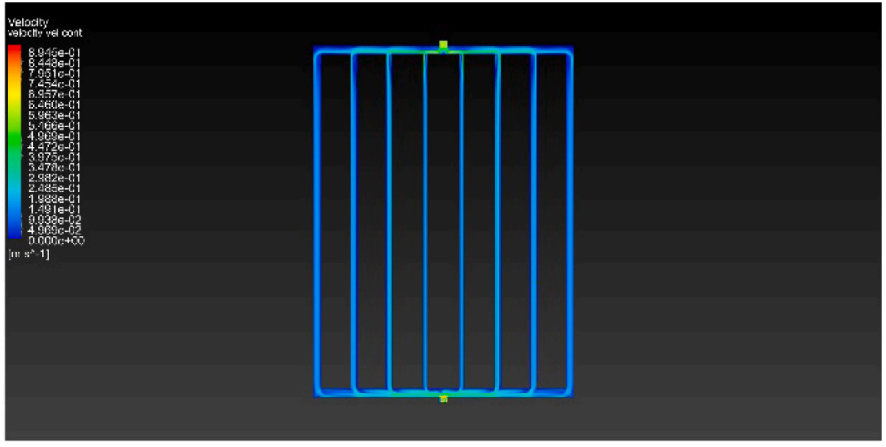

(b)

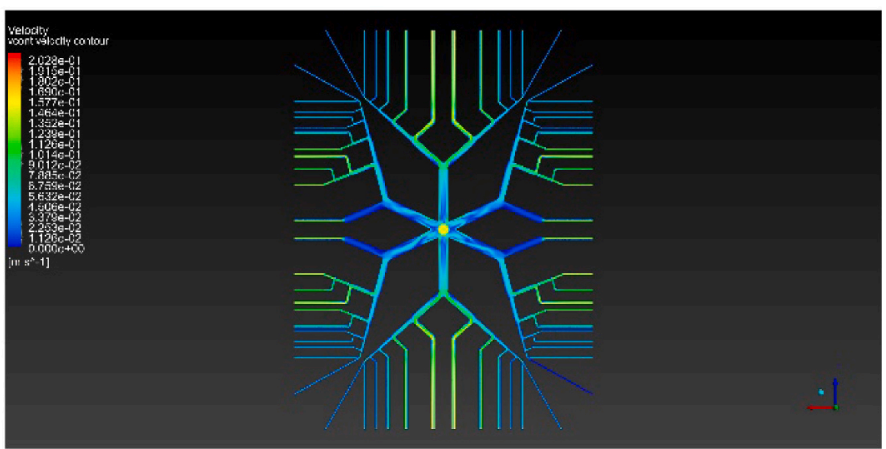

(d)

Fig. 3. Velocity distribution contours in the tubes for laminar flow ( $R e=1000-1200)$ : (a) serpentine configuration, (b) parallel flow tubes configuration, (treeshaped flow configuration (inlet at edge), and (d) tree-shaped flow configuration (inlet at center) designed with allometric laws for laminar flow. 


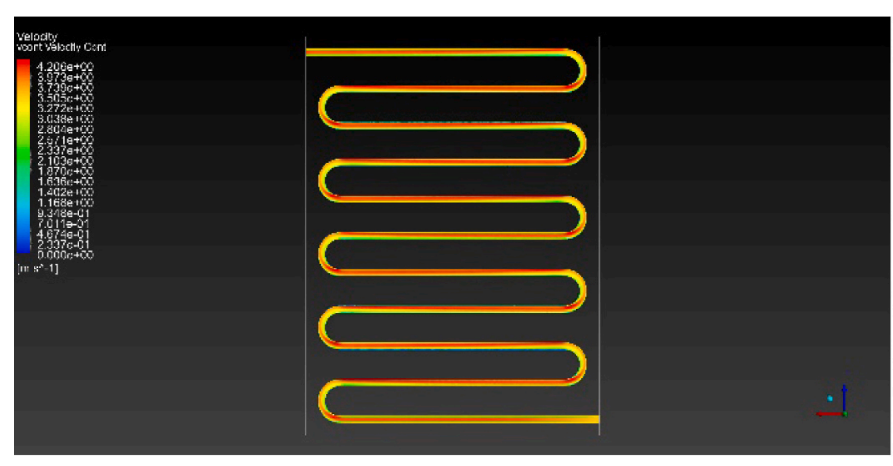

(a)

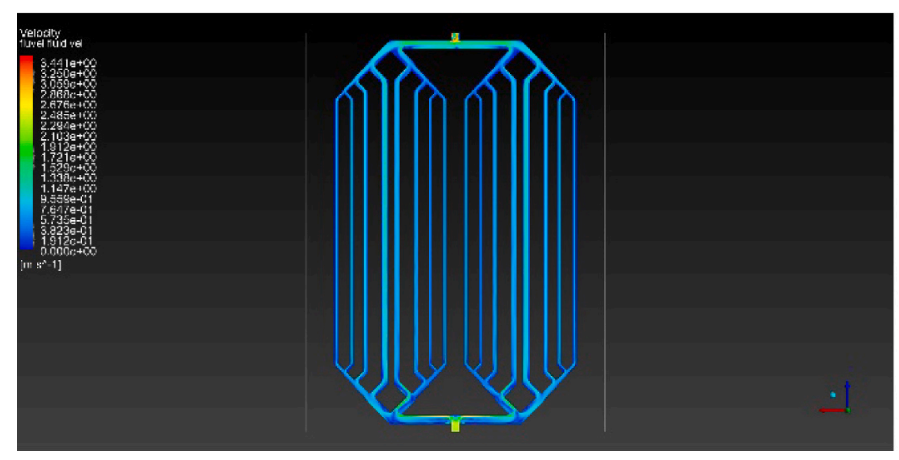

(c)

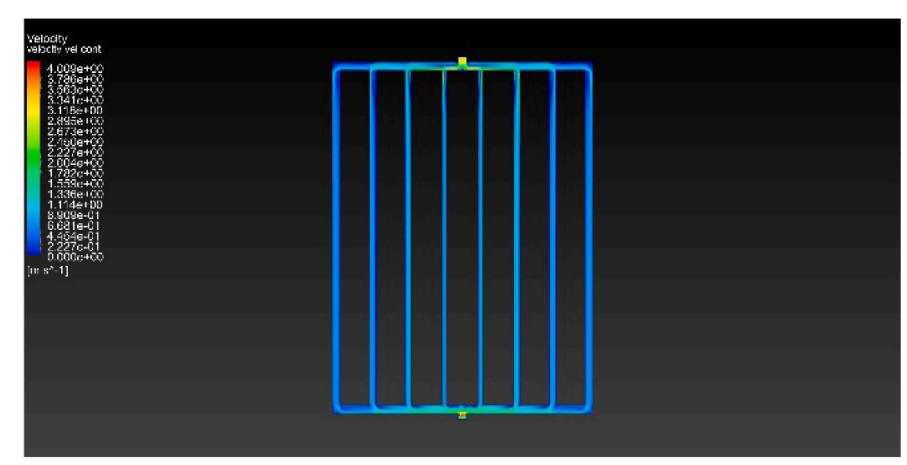

(b)

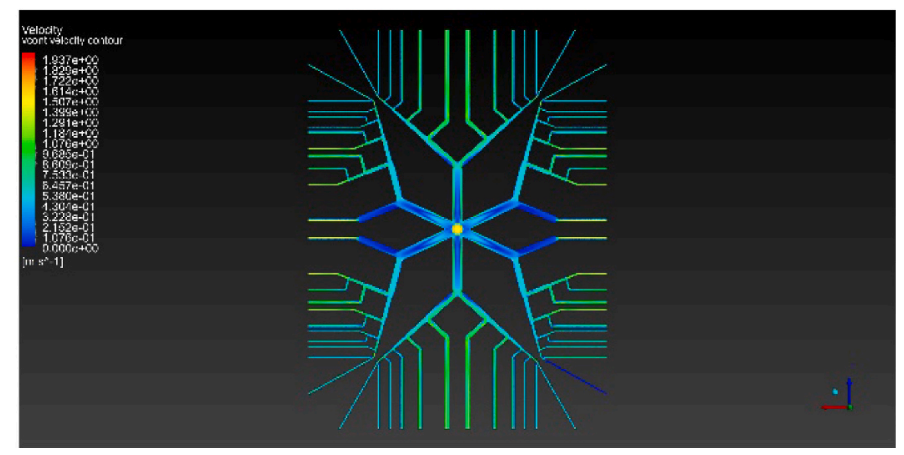

(d)

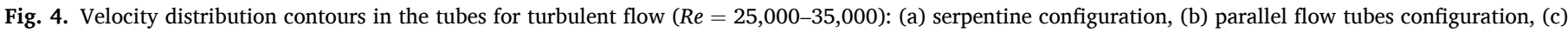
tree-shaped flow configuration (inlet at edge), and (d) tree-shaped flow configuration (inlet at center) designed with allometric laws for laminar flow.

sharp angles, the parallel design is more susceptible to flow separation (Fig. 5). Flow separation in the tubes may create recirculation which affect the heat transfer.

The configuration depicted at Fig. $1 \mathrm{~b}$ is formed by parallel tubes of different diameters, but the tubes on the left side of the inlet are a perfect reflection of the right side of the inlet. Comparing the flows of the tubes

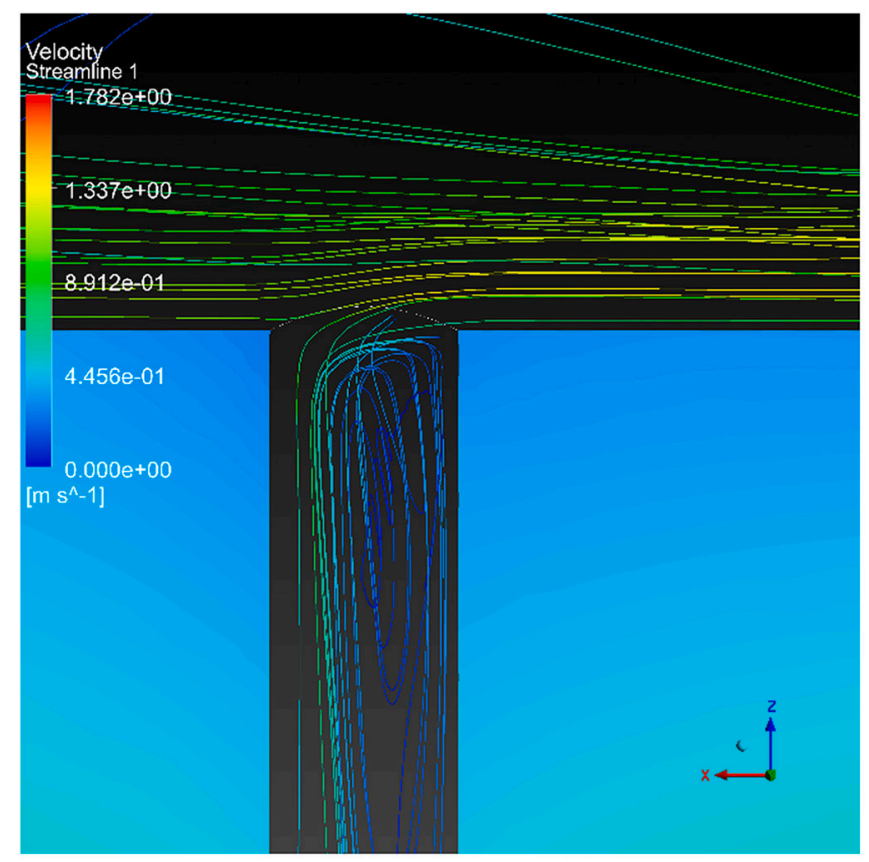

Fig. 5. Recirculation in the parallel tube configuration (Fig. 1b) for $R e=1000$. on the left and on the right sides we conclude that the flow distribution is heterogeneous for both flow regimes. A feature known and explained by different authors (see for example, Andrade et al. [27] and Pepe et al. [28]) is that asymmetric flow occurs in symmetric branched structures in laminar flow regime. The distribution of flow heterogeneity is more significant for higher Reynolds numbers. Regarding the tree flow configuration with inlet at edge (Figs. 3c,d and 4c,d), the flow distribution is more heterogeneous in the tubes with larger diameter (at the first levels of bifurcation). As the diameter of the tubes decreases, the flow asymmetry is reduced, being practically symmetrical for the smaller tubes under laminar flow. However, for turbulent regime there is some heterogeneity in the flow distribution even in the smallest tubes. This is especially observable at Fig. $4 d$ at the 4th to 6th level of bifurcation. The results illustrated in Figs. 3c,d and 4c,d agree with the findings of Andrade et al. [27].

Figs. 6 and 7 show the temperature distribution in the cooled plate for laminar and turbulent flow regimes. The patterns of temperature distributions vary at different Reynolds numbers. Under laminar flow, the average temperature of the plates are higher than that of the plates under turbulent flow regime. As a result, parallel flow tubes configuration, serpentine configuration, and tree-shaped flow configuration (inlet at edge) show significant hot spots on considerable extensions of the plate under laminar flow regime. There are extensive areas with higher temperatures, namely in the central area of the plates, exception for the configuration shown in Fig. $6 \mathrm{~d}$ where the hot spots are in the corners.

For turbulent regime, hot spots appear on the edges of the plates for serpentine and tree-shaped flow configurations (inlet at edge). Plates have a more uniform temperature when cooled by a tree-shaped flow configuration with inlet at center (laminar flow), and by parallel tubes (turbulent flow). Besides, the average temperature of the plates with the tree configurations (Fig. 7c and d) is substantially lower than that of the other configurations. Note that the configuration in Fig. 1d was designed 


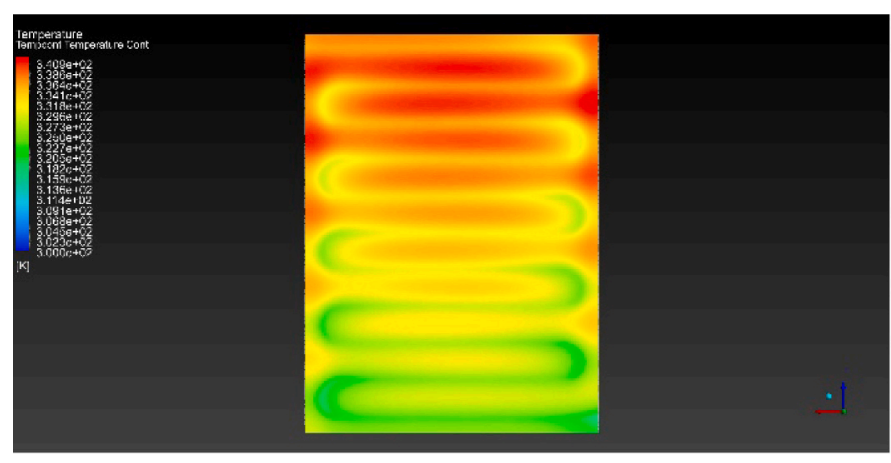

(a)

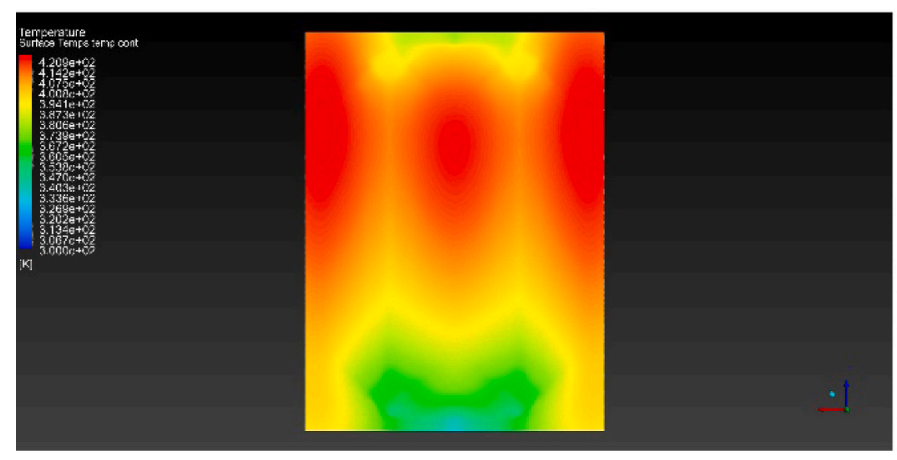

(c)

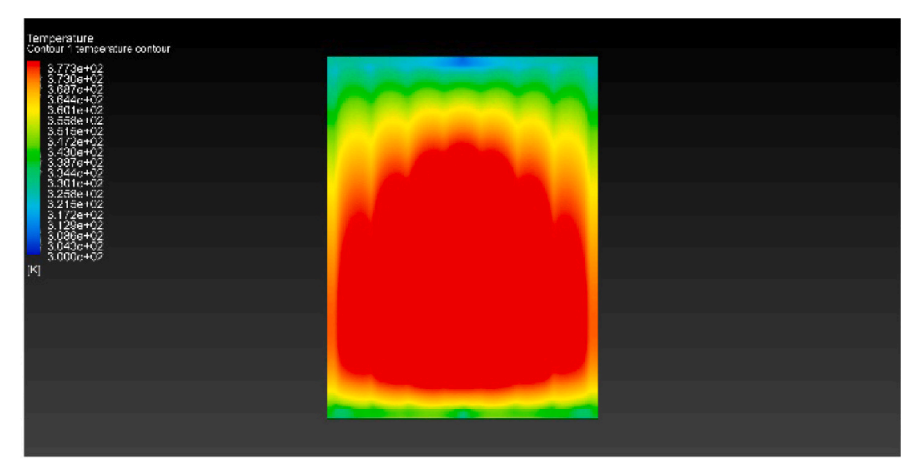

(b)

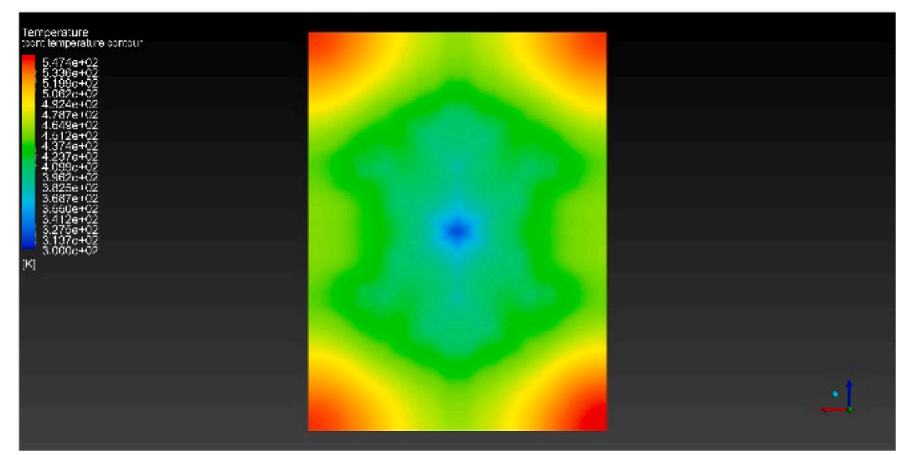

(d)

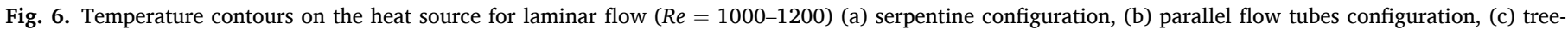
shaped flow configuration (inlet at edge), and (d) tree-shaped flow configuration (inlet at center) designed with allometric laws for laminar flow.

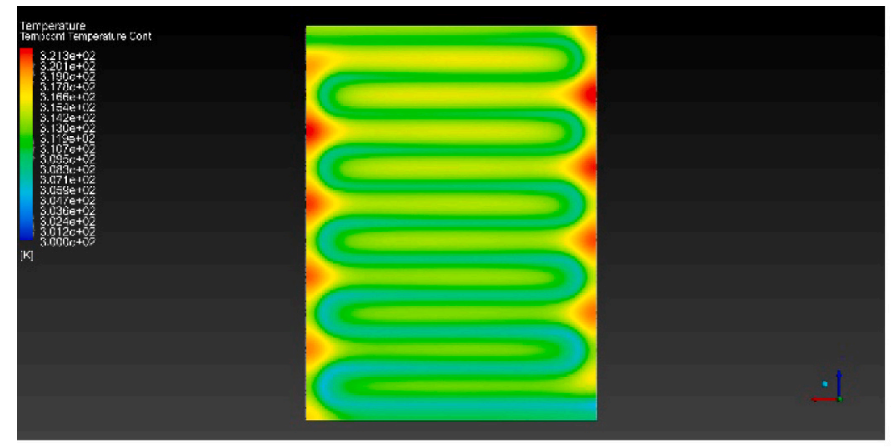

(a)

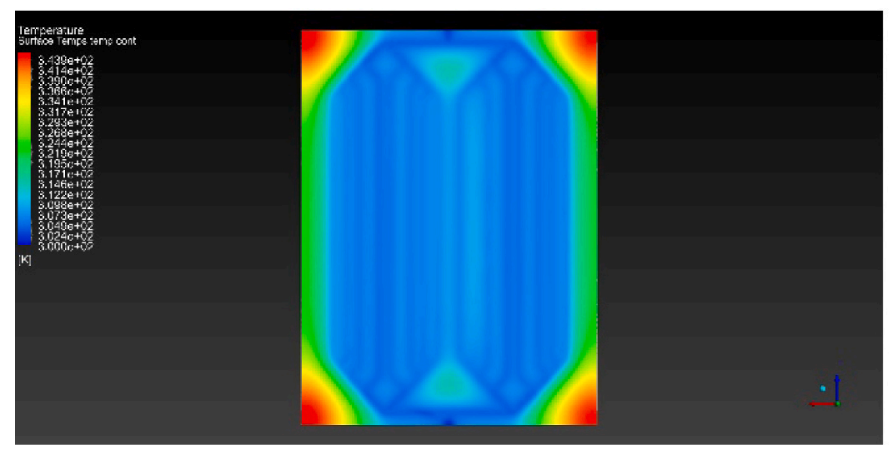

(c)

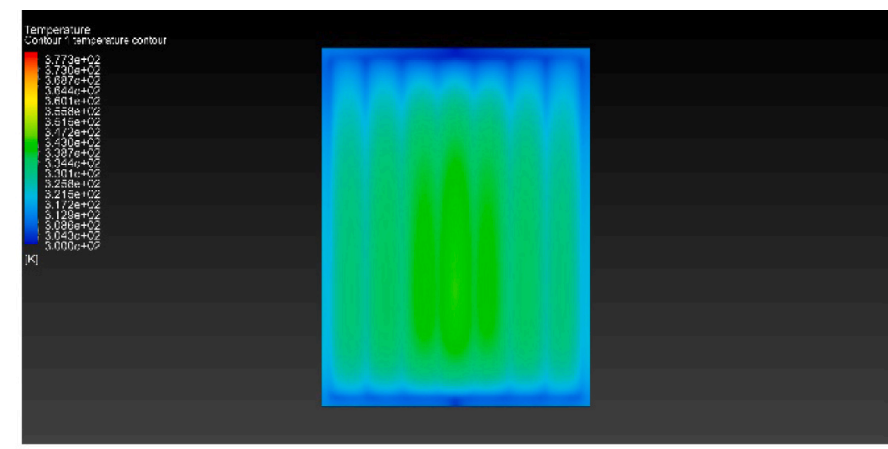

(b)

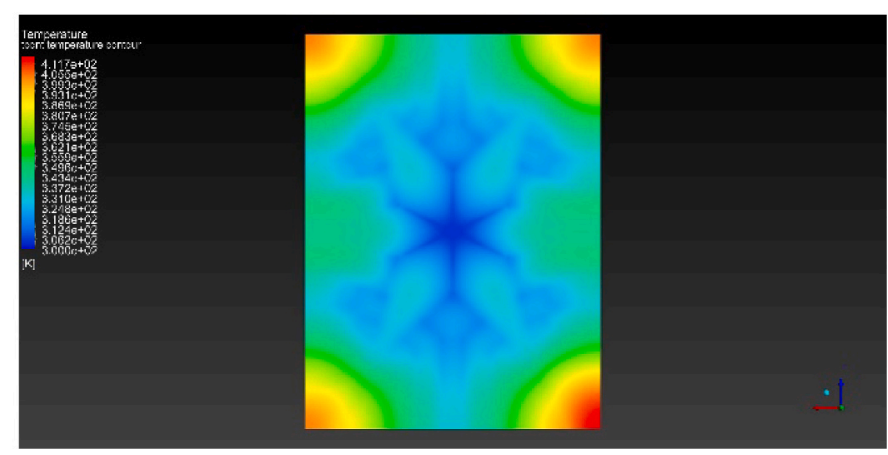

(d)

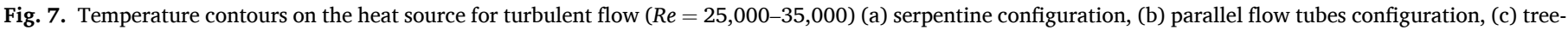
shaped flow configuration (inlet at edge), and (d) tree-shaped flow configuration (inlet at center) designed with allometric laws for laminar flow. 
with the optimal allometric laws for laminar flow regime, but in turbulent flow regime it continues to perform well, with the plate having one of the lowest average temperatures (Fig. 7).

The temperature of the plates is not homogeneous. Maximum and minimum temperatures recorded on the plates, and average plate temperatures are presented in Figs. 8 to 10. The results are presented in terms of dimensionless temperatures defined as the ratio between the plate temperature and inlet fluid temperature. Reported in the figure is that, as expected, the maximum, minimum and average temperatures decrease with the Reynolds number. The highest and lowest values of maximum temperatures occur for plates cooled with a tree-shaped configuration (inlet at center) and with a serpentine configuration, respectively (Fig. 8). While for a serpentine almost half of the plate surface is under high temperature, for a plate with a tree-shaped configuration the zones of high temperature are restricted to portions close to the corners (Fig. 6). Notice that high temperature zones result of less plate coverage per tubes, and less access to the fluid to remove heat.

It is interesting to note that with respect to minimum temperature, a plate with a serpentine configuration presents the highest values and a plate with a tree-shaped configuration (inlet at center) the lowest values (Fig. 9). The surface of plate with the minimum temperatures is located close to the inlet (Fig. 6). For a plate with a serpentine, these temperatures occur along one edge of the plate but for a plate with the tree configuration (inlet at center) it covers the central portion of the plate. This result reveals the importance of the inlet position.

The average temperature of the plate seems to be less sensitive to the design for heat sinks, particularly for very high Reynolds numbers (Fig. 10). Reported in the figure is that the plate with a serpentine has the lowest average temperature in the entire range of Reynolds numbers studied, while the plate with parallel tubes has the highest values. It is worth noting that for the higher range of $R e$ values, average temperature of plates with serpentine and tree-shaped configuration (inlet at center) are identical.

\subsection{Thermal and fluid flow performances}

Here the effect of different configuration of tubes on heat dissipated, and flow resistance is analyzed based on Eqs. (10), (11) and (14). The thermal performance (Eq. (10)) of the flow configuration is illustrated at (Fig. 11). Reported in this figure is the increase of thermal performance with the Reynolds number. This figure shows that the parallel tube design has the lowest performance (i.e., less heat dissipation) for all range of Reynolds numbers. Besides, the tree-shaped design of the tubes (inlet at center of the plate) has the best performance for Reynolds numbers up to $10^{4}$ while the serpentine design has the best performance

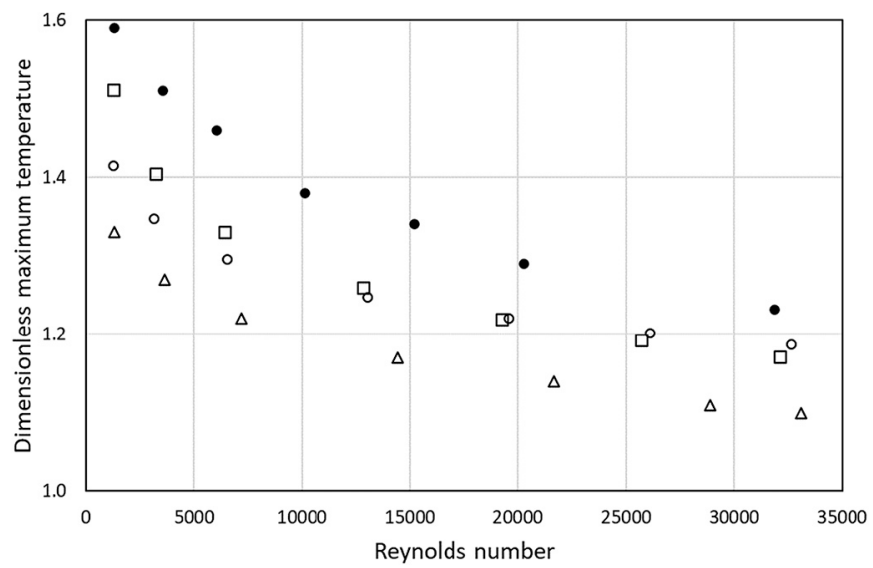

Fig. 8. Dimensionless maximum temperature obtained in the plate vs Reynolds Number for cooling configurations depicted at Fig. 1: $\Delta$ serpentine, $\square$ parallel flow tubes, o tree-shaped flow configuration (inlet at edge), $\bullet$ tree-shaped flow configuration (inlet at center) designed with allometric laws for laminar flow.

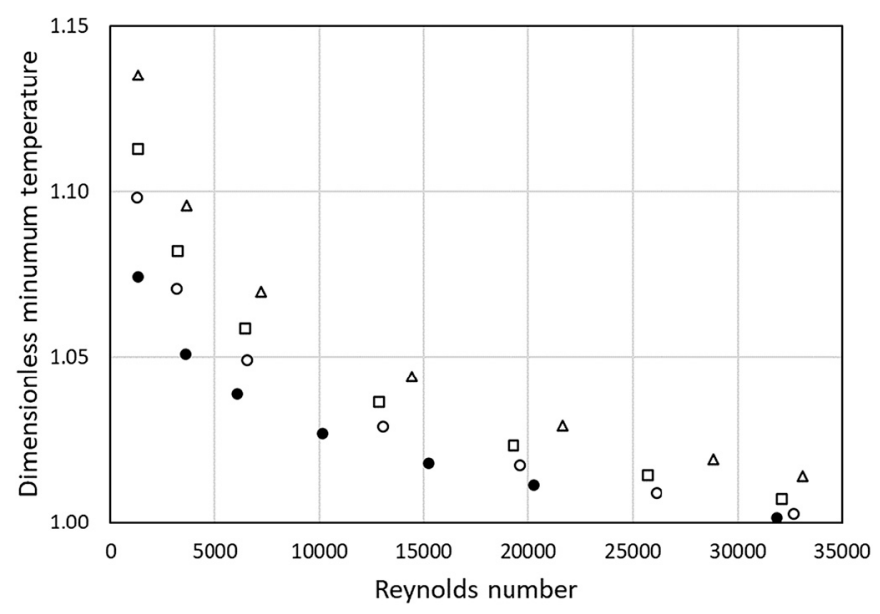

Fig. 9. Dimensionless minimum temperature obtained in the plate vs Reynolds Number for cooling configurations depicted at Fig. 1: $\Delta$ serpentine, $\square$ parallel flow tubes, o tree-shaped flow configuration (inlet at edge), $\bullet$ tree-shaped flow configuration (inlet at center) designed with allometric laws for laminar flow.

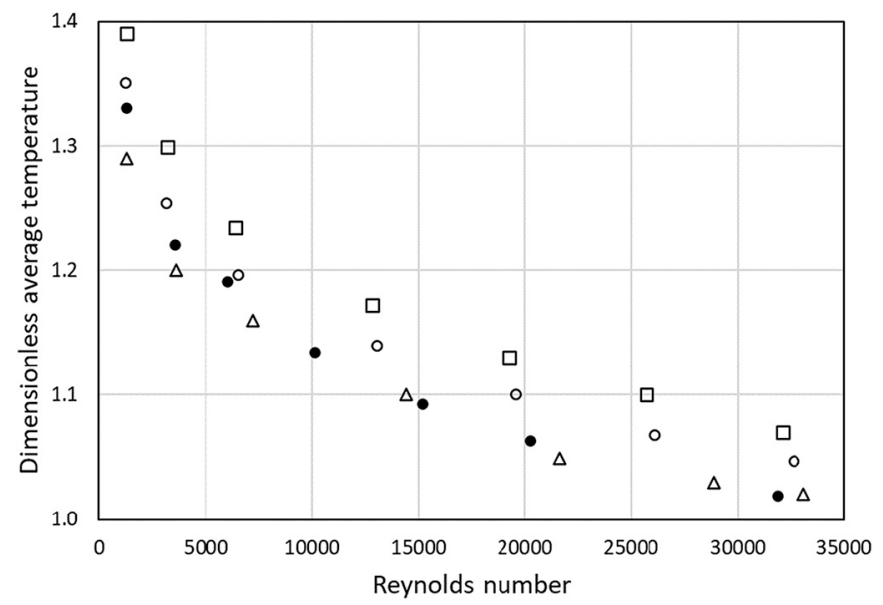

Fig. 10. Dimensionless average plate temperature vs Reynolds Number for cooling configurations depicted at Fig. 1: $\Delta$ serpentine, $\square$ parallel flow tubes, o tree-shaped flow configuration (inlet at edge), • tree-shaped flow configuration (inlet at center) designed with allometric laws for laminar flow.

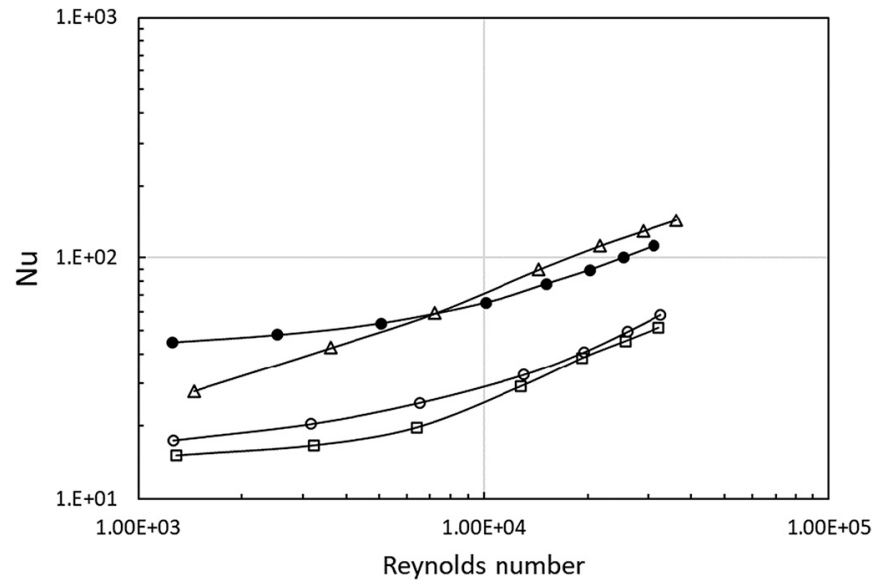

Fig. 11. Thermal performance $N u$ vs Reynolds Number for cooling configurations depicted at Fig. 1 (a higher $N u$ is better): $\Delta$ serpentine, $\square$ parallel flow tubes, o tree-shaped flow configuration (inlet at edge), - tree-shaped flow configuration (inlet at center) designed with allometric laws for laminar flow. 
for higher values of Reynolds numbers. Note that the tree design with inlet in the center is built with the optimal geometric relations for laminar flow regime. But even despite this, it has a meritorious thermal performance for transition and turbulent flows.

Fig. 12 presents the variation of the hydraulic performance (Eq. (11)) against Reynolds number. Reported in the figure is that the hydraulic performance decreases with Reynolds number. For the range of Reynolds numbers studied, it is worth noting that the tree design with inlet in the center of the plate has the best hydraulic performance and the serpentine design shows clearly the worst one. In summary, it is particularly remarkable that the tree configuration with inlet in the center holds the best performance in the entire range of Re analyzed, which shows the robustness of the design even in outside of the laminar flow.

The definition of the hydrothermal performance depends on the constraints of the application. Consider that the focus is only on the combined effect of dissipated heat and pressure drop. Fig. 13 shows the ratio of $\mathrm{Nu}$ to $\mathrm{Eu}$ against the Reynolds number. It must be noted that $\mathrm{Nu}$ / $\mathrm{Eu}$ increases with the Reynolds number $\left(\frac{N u}{E u}=\frac{16}{\pi^{2} \rho D^{3} k_{f} A_{l s}} \frac{Q_{\text {hea }} \dot{m}_{f}^{2}}{T^{*} \Delta p}=\frac{\mu^{2}}{\rho D k_{f} A_{l s}} \frac{Q_{\text {heat }} R e^{2}}{T^{*} \Delta p}\right)$. Reported in the figure is also that the performance based on $\mathrm{Nu} / \mathrm{Eu}$ is best for the tree design with inlet in the center of the plate in the entire range of Reynolds numbers studied. It is worth noting that the tree design (edge inlet) and parallel tubes show similar performances and the serpentine design shows clearly the worst performance.

Alternatively, the hydrothermal performance is presented in Fig. 14 as plot of $\zeta$ defined by Eq. (14) against Reynolds number. Here the performance is defined in terms of dissipated heat and pumping power, and the plot shows that $\zeta$ decreases with Reynolds number $\left(\zeta=\frac{\mu^{2}}{c_{p . f} \rho^{2} A_{p}} \frac{Q_{\text {heat }}}{\bar{T}_{p} P}=\frac{16}{\pi^{2} c_{p . f} \rho^{2} A_{p} D^{2}} \frac{\dot{m}^{2} Q_{\text {heat }}}{\bar{T}_{p} P R e^{2}}\right)$. It is worth mentioning that the tree-shaped configuration with center inlet shows the best performance over the entire range of Reynolds numbers studied whereas the serpentine configuration has the lowest performance. Reported in this figure is also that, as for $\mathrm{Nu} / \mathrm{Eu}$, the tree design (edge inlet) and parallel tubes show similar performances.

In summary, regardless of the calculation form, Figs. 13 and 14 show that the tree-shaped configuration with center inlet has the best performance over the entire range of Reynolds numbers studied, and the serpentine configuration has the lowest performance among all the

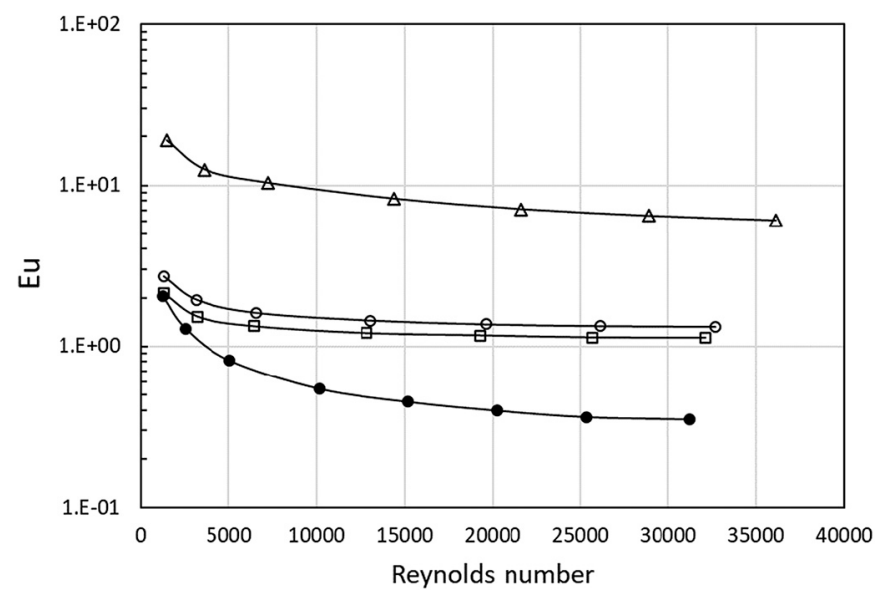

Fig. 12. Hydraulic performance $E u$ vs Reynolds Number for cooling configurations depicted at Fig. 1 (a lower $E u$ is better): $\Delta$ serpentine, $\square$ parallel flow tubes, o tree-shaped flow configuration (inlet at edge), • tree-shaped flow configuration (inlet at center) designed with allometric laws for laminar flow.

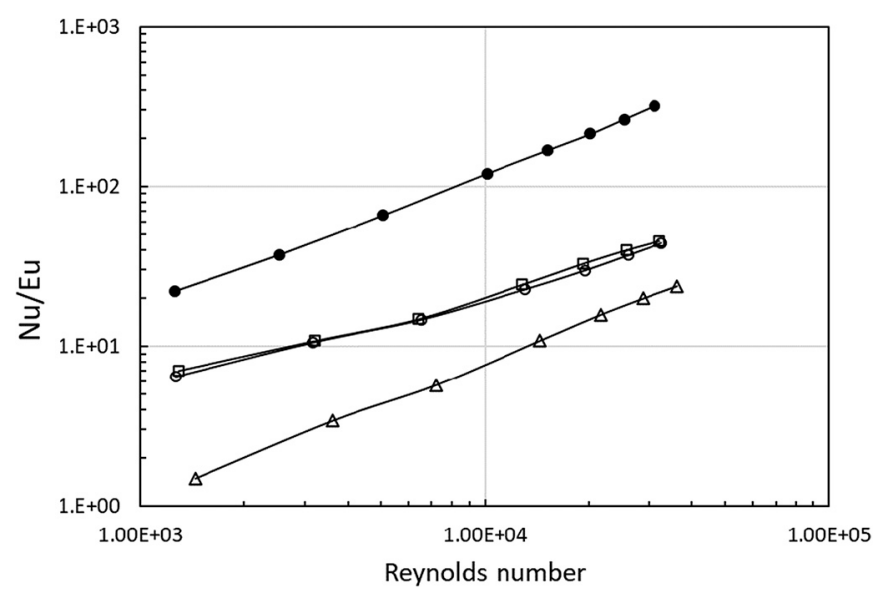

Fig. 13. Ratio of Nusselt to Euler numbers vs Reynolds Number for cooling configurations depicted at Fig. 1 (a higher $\mathrm{Eu} / \mathrm{Nu}$ is better): $\Delta$ serpentine, $\square$ parallel flow tubes, o tree-shaped flow configuration (inlet at edge), • treeshaped flow configuration (inlet at center) designed with allometric laws for laminar flow.

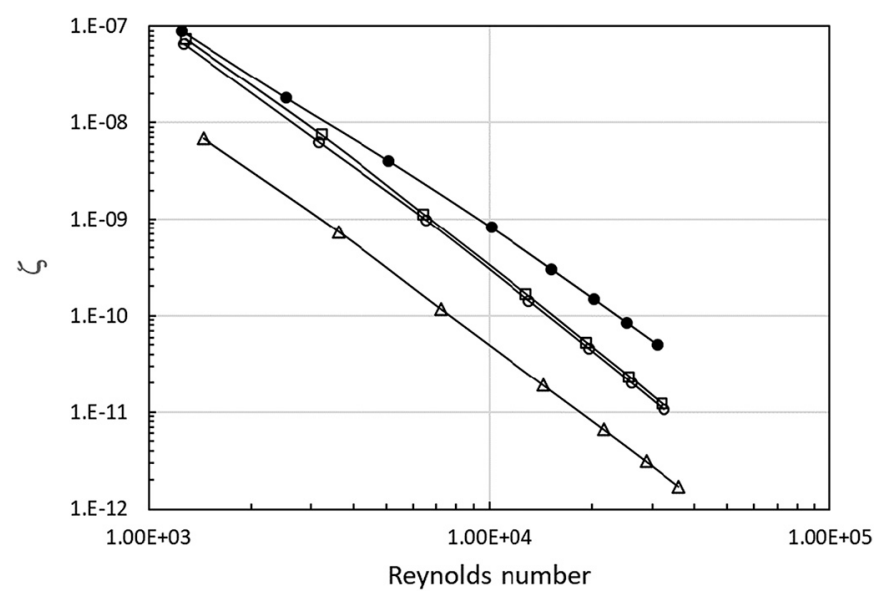

Fig. 14. Performance $\zeta$ (Eq. (14)) vs Reynolds Number for cooling configurations depicted at Fig. 1 (a higher $\zeta$ is better): $\Delta$ serpentine, $\square$ parallel flow tubes, o tree-shaped flow configuration (inlet at edge), • tree-shaped flow configuration (inlet at center) designed with allometric laws for laminar flow.

\section{designs.}

The results depicted at Figs. 11 to 14 can be fitted by suitable mathematical expressions and the parameters of these equations determined. A power-law model best fits the data obtained

$Y=a_{Y} R e^{b_{Y}}$

where the dependent variable $\mathrm{Y}$ is $\mathrm{Nu}, \mathrm{Eu}, \mathrm{Nu} / \mathrm{Eu}$ and $\zeta$, and $\mathrm{a}_{\mathrm{Y}}$ and $\mathrm{b}_{\mathrm{Y}}$ are unknown constants. These constants are determined using the least squares method (Table 5) over the Reynolds number range of $10^{3}$ to 3.5 $\times 10^{4}$. The coefficient of determination is greater than 0.9 .

\section{Conclusions}

A CFD study is conducted to investigate the performance of several configurations of tubes that can be used for cooling high heat-generating systems. Size limitations are included in this study (the volume of the panel and the total lateral surface area of tubes are constants), and the study is performed to investigate the cooling performance of plates with 4 different configurations of tubes.

The results show that the average temperature of the plate is slightly dependent of cooling flow design, but depends on the Reynolds number. 
Table 5

Non-linear regression model $Y=a_{Y} R e^{b_{Y}}$.

\begin{tabular}{llll}
\hline Thermal performance $(\mathrm{Nu})$ & & & \\
\hline Flow configuration & $\mathrm{a}_{\mathrm{Nu}}$ & $\mathrm{b}_{\mathrm{Nu}}$ & $\mathrm{r}^{2}$ \\
\hline Serpentine & 0.6189 & 0.5189 & 0.998 \\
Parallel tubes & 0.6926 & 0.4048 & 0.956 \\
Tree (inlet at edge) & 1.1026 & 0.3688 & 0.946 \\
Tree (inlet at center) & 5.0749 & 0.2887 & 0.944
\end{tabular}

\begin{tabular}{|c|c|c|c|}
\hline \multicolumn{4}{|c|}{ Hydraulic performance $(E u)$} \\
\hline Flow configuration & $\mathrm{a}_{\mathrm{Eu}}$ & $\mathrm{b}_{\mathrm{Eu}}$ & $\mathrm{r}^{2}$ \\
\hline Serpentine & 221.72 & -0.344 & 0.991 \\
\hline Parallel tubes & 7.4461 & -0.188 & 0.928 \\
\hline Tree (inlet at edge) & 11.426 & -0.214 & 0.944 \\
\hline Tree (inlet at center) & 100.50 & -0.557 & 0.993 \\
\hline
\end{tabular}

\begin{tabular}{llll}
\hline $\mathrm{Nu} / \mathrm{Eu}$ & & & \\
\hline Flow configuration & $\mathrm{a}_{\mathrm{Nu} / \mathrm{Eu}}$ & $\mathrm{b}_{\mathrm{Nu} / E u}$ & $\mathrm{r}^{2}$ \\
\hline Serpentine & 0.0028 & 0.8631 & 0.999 \\
Parallel tubes & 0.0930 & 0.5926 & 0.995 \\
Tree (inlet at edge) & 0.0937 & 0.5850 & 0.993 \\
Tree (inlet at center) & 0.0555 & 0.8336 & 0.999 \\
& & & \\
\hline$\zeta$ defined by Eq. (14) & & & \\
\hline Flow configuration & $\mathrm{a} \zeta$ & $\mathrm{b}_{\mathrm{N}} \zeta$ & $\mathrm{r}^{2}$ \\
\hline Serpentine & 1.1301 & -2.590 & 0.999 \\
Parallel tubes & 24.349 & -2.722 & 0.999 \\
Tree (inlet at edge) & 15.166 & -2.685 & 0.999 \\
Tree (inlet at center) & 1.4927 & -2.322 & 0.999 \\
\hline
\end{tabular}

Temperature uniformity of the plates is strongly dependent on both the cooling flow design and Reynolds number. Turbulent flows through the tubes cause lower average temperature of plates, and more uniform temperatures across plates. In general, for laminar flow plates present extensive areas of high temperature, with the exception of the plate with a tree-shaped configuration where the zones of high temperature are restricted to small areas close to the corners.

The thermal performance of the plates increases with Reynolds number but the hydraulic performance decreases with Reynolds number (increase the pressure drop penalty). The definition of the hydrothermal performance depends on the constraints of the application. So, this study presents two ways to calculate the hydrothermal performance. In both cases, the best performance is obtained for the plate with a tree-shaped network of tubes with the inlet placed at the center of the plate. The performances of the plates with parallel flow tubes configuration and tree-shaped flow configuration with inlet at edge are very similar. The lower performance is obtained when plate cooling is done using a serpentine tube.

The best solution in terms of temperatures (lower average plate temperature, more uniform plate temperature) and hydrothermal performance is obtained for a plate with a tree-shaped network of tubes with the inlet placed at the center of the plate in the entire range of Reynolds numbers studied.

The results obtained in this study will provide important insights for the designers to improve hydrothermal management of cooling plates which are widely used from electric vehicle power batteries to photovoltaic solar panels.

\section{Authors' contributions}

S. C. performed the CFD simulations, analyzed the results and designed the figs. A.F.M. and M. A. are involved in planning, supervised the work and contributed to the interpretation of the results, and wrote the manuscript with input from S. C. A.F.M. conceived of the idea of the project. All authors provided critical feedback and helped shape the research, analysis and manuscript.

\section{Declaration of Competing Interest}

The authors declare that they have no known competing financial interests or personal relationships that could have appeared to influence the work reported in this paper.

\section{Acknowledgements}

A.F.M. work is framed within the activities of the Portuguese funding awarded by FCT - Foundation for Science and Technology, I.P., projects UIDB/04683/2020 and UIDP/04683/2020.

\section{References}

[1] Y. Li, L. Gong, B. Ding, M. Xu, Y. Joshi, Thermal management of power electronics with liquid cooled metal foam heat sink, Int. J. Therm. Sci. 163 (2021) 106796.

[2] K. Kirad, M. Chaudhari, Design of cell spacing in lithium-ion battery module for improvement in cooling performance of the battery thermal management system, J. Power Sources 481 (2021) 229016.

[3] J. Chen, S. Kang, J.E.Z. Huang, K. Wei, B. Zhang, H. Zhu, Y. Deng, F. Zhang, G. Liao, Effects of different phase change material thermal management strategies on the cooling performance of the power lithium ion battery system: a review, J. Power Sources 442 (2019) 227228.

[4] Y. Jia, G. Alva, G. Fang, Development and applications of photovoltaic-thermal systems: a review, Renew. Sust. Energ. Rev. 102 (2019) 249-265.

[5] W. He, T.T. Chow, J. Ji, J. Lu, G. Pei, L.-S. Chan, Hybrid photovoltaic and thermal solar collector designed for natural circulation of water, Appl. Energy 83 (2006) 199-210.

[6] Y. Fan, P.S. Lee, L.-W. Jin, B.W. Chua, Experimental investigation on heat transfer and pressure drop of a novel cylindrical oblique fin heat sink, Int. J. Therm. Sci. 76 (2014) 1-10.

[7] H. Zhang, X. Liu, Numerical analysis of the flow and heat transfer characteristics in serpentine microchannel with variable bend amplitude, Int. J. Numer. Method. H. 06 (2020) 0334.

[8] J. Wang, S. Lu, Y. Wang, C. Li, K. Wang, Effect analysis on thermal behavior enhancement of lithium-ion battery pack with different cooling structures, J. Energy Stor. 32 (2020) 101800.

[9] C. Leng, X. Wang, T. Wang, An improved design of double-layered microchannel heat sink with truncated top channels, Appl. Therm. Eng. 79 (2015) 54-62.

[10] S.M. Senn, D. Poulikakos, Laminar mixing, heat transfer and pressure drop in treelike microchannel nets and their application for thermal management in polymer electrolyte fuel cells, J. Power Sources 130 (2004) 178-191.

[11] Y. Chen, C. Zhang, M. Shi, Y. Yang, Thermal and hydrodynamic characteristics of constructal tree-shaped minichannel heat sink, AICHE J. 2010 (2018-2029) 56.

[12] M. Mosa, M. Labat, S. Lorente, Role of flow architecture on the design of radiant cooling panels, a Constructal approach, Appl. Therm. Eng. 150 (2019) 1345-1352.

[13] M. Mosa, M. Labat, S. Lorente, Constructal design of flow channels for radiant cooling panels, Int. J. Therm. Sci. 145 (2019) 106052.

[14] Z. Lu, K. Zhang, Study on the performance of a Y-shaped liquid cooling heat sink based on constructal law for electronic chip cooling, J. Therm. Sci. Eng. Appl. 13 (2021), 034501.

[15] C. Shen, F. Liu, S. Qiu, X. Liu, F. Yao, Y. Zhang, Numerical study on the thermal performance of photovoltaic thermal (PV/T) collector with different parallel cooling channels, Sustain. Energy Technol. Assess. 45 (2021) 101101.

[16] S.S. Joshi, A.S. Dhoble, Photovoltaic-thermal systems (PVT): technology review and future trends, Renew. Sust. Energ. Rev. 92 (2018) 848-882.

[17] G. Narendran, N. Gnanasekaran, D.A. Perumal, A review on recent advances in microchannel heat sink configurations, Recent Patents Mech. Eng. 11 (2018) 190-215.

[18] A. Bejan, S. Lorente, Design with Constructal Theory, John Wiley \& Sons, Hoboken, USA, 2008.

[19] W. Wechsatol, S. Lorente, A. Bejan, Tree-shaped insulated designs for the uniform distribution of hot water over an area, Int. J. Heat Mass Transf. 44 (2001) 3111-3123.

[20] A.F. Miguel, Towards methodologies for optimal fluid networks design, J. Appl. Fluid Mech. 12 (2019) 1223-1229.

[21] A.F. Miguel, Optimal Y-shaped constructs heat sinks under different size constraints, Int. J. Heat Mass Transf. 131 (2019) 64-71.

[22] A.F. Miguel, A study of entropy generation in tree-shaped flow structures, Int. J. Heat Mass Transf. 92 (2016) 349-359.

[23] I.B. Celik, U. Ghia, P.J. Roache, C.J. Freitas, H. Coleman, P.E. Raad, Procedure for estimation and reporting of uncertainty due to discretization in CFD applications, J. Fluids Eng. 130 (2008), 078001.

[24] A.F. Miguel, L.A.O. Rocha, Tree-Shaped Fluid Flow and Heat Transfer, Springer, NY, USA, 2018.

[25] A.F. Miguel, Occlusions in dendritic flow networks, Physica A 535 (2019) 122473. 
[26] ANSYS Fluent Theory Guide, ANSYS, Inc., 275 Technology Drive Canonsburg, PA 15317, 2013.

[27] J.S. Andrade Jr., A.M. Alencar, M.P. Almeida, J. Mendes Filho, S.V. Buldyrev, S. Zapperi, H.E. Stanley, B. Suki, Asymmetric flow in symmetric branched structures, Phys. Rev. Lett. 81 (1998) 926-929.
[28] V.R. Pepe, L.A.O. Rocha, A.F. Miguel, Optimal branching structure of fluidic networks with permeable walls, Biomed. Res. Int. 2017 (2017) 5284816. 